\title{
Fleuves, océans, ports et navires: stratégie(s) et imaginaire maritimes au Québec
}

\section{Alessia Vignoli}

\section{(2) OpenEdition}

1 Journals

\section{Édition électronique}

URL : https://journals.openedition.org/studifrancesi/32443

DOI : 10.4000/studifrancesi.32443

ISSN : 2421-5856

Éditeur

Rosenberg \& Sellier

\section{Édition imprimée}

Date de publication : 1 août 2020

Pagination : 457-458

ISSN : 0039-2944

\section{Référence électronique}

Alessia Vignoli, «Fleuves, océans, ports et navires: stratégie(s) et imaginaire maritimes au Québec », Studi Francesi [En ligne], 191 (LXIV | II) | 2020, mis en ligne le 01 septembre 2020, consulté le 18 septembre 2021. URL : http://journals.openedition.org/studifrancesi/32443 ; DOI : https://doi.org/10.4000/ studifrancesi.32443

Ce document a été généré automatiquement le 18 septembre 2021.

\section{(c)}

Studi Francesi è distribuita con Licenza Creative Commons Attribuzione - Non commerciale - Non opere derivate 4.0 Internazionale. 


\title{
Fleuves, océans, ports et navires: stratégie(s) et imaginaire maritimes au Québec
}

\author{
Alessia Vignoli
}

\section{RÉFÉRENCE}

Fleuves, océans, ports et navires: stratégie(s) et imaginaire maritimes au Québec, "Ponti/Ponts. Langues, littératures et civilisations des pays francophones" 18, 2018, 282 pp.

1 Le numéro 18 de la revue Ponti/Ponts propose un ensemble d'études consacrées à une seule aire francophone, le Québec, et à un aspect particulier: l'imaginaire maritime, dominé par la présence du fleuve Saint-Laurent, «[...] élément-clé de l'espace national et du paysage culturel québécois» (p. 7). La première contribution présentée est celle de François-Régis FOURNIER, Le Saint-Laurent, source et défi, vie et survie, centrée sur le rôle du fleuve dans la vie des habitants du Québec, abordé à partir d'une perspective historique. Fournier développe sa thèse, selon laquelle les Québécois «[...] ont dû apprivoiser l'ensemble du territoire en parcourant le fleuve pour y survivre d'abord, puis pour y vivre et y bien vivre ensuite» (p. 15), en traçant une sorte d'historique du rapport au fleuve, de l'arrivée des premiers explorateurs européens au $\mathrm{xVI}^{\mathrm{e}}$ siècle jusqu'à l'époque contemporaine.

2 Dans D'un océan à l'autre: spatialité de la parole et lisibilité des lieux chez Jacques Poulin, Franca BRUERA et Valeria MARINO analysent le rôle des cartes géographiques dans Volkswagen Blues de Jacques Poulin. L'objectif des auteures est de montrer que la présence des cartes dans le texte n'a pas seulement une fonction de rappel des déplacements des deux personnages, Jack et Pitsémine, mais, à un niveau plus profond, elle est ancrée à la structure de l'intrigue. Les auteures désirent mettre en évidence l'aspect ambivalent du dispositif cartographique utilisé par Poulin, où le fleuve joue un rôle de premier plan. Afin de montrer le rapport entre les fleuves et le roman, elles 
expliquent que c'est au long des cours d'eau que les personnages se racontent, en développant des métarécits, c'est-à-dire «[...] des histoires brèves et des digressions situées dans le cadre de l'histoire principale» (p. 33). Le Saint-Laurent joue le rôle de catalyseur de la mémoire de Jack, mais Pitsémine aussi est emportée émotivement par le paysage fluvial. Un autre fleuve emblématique de l'espace nord-américain, le Mississippi, déclenche des émotions fortes chez les protagonistes, à cause de son caractère mythique. En conclusion, Bruera et Marino constatent que, dans Volkswagen Blues, «[...] les cartes ont été mélangées et confondues jusqu’à créer un paysage métis, hybride et conforme à celui qui a été représenté à l'époque où le livre a été conçu» (p. 40), un paysage où la culture et les identités ont un caractère pluriel et transfrontalier.

3 Nadine VINCENT, dans Habiter le Saint-Laurent. Présence du fleuve dans les dictionnaires faits au Québec de 1880 à aujourd'hui, illustre le rôle du Saint-Laurent dans la mémoire collective québécoise à partir des occurrences associées au fleuve dans trois dictionnaires. Les comparaisons entre les définitions données par les dictionnaires à travers les siècles permettent d'observer que chaque dictionnaire «[...] raconte la vie du fleuve et de ses habitants à un moment précis» (p. 46). Dans son analyse, Vincent parvient à montrer qu'«[...] un dictionnaire de langue, plus qu'un outil normatif, est avant tout le miroir d'une société à un moment déterminé» (p. 60).

4 La contribution de Maura FELICE, Le voyage de la demoiselle de François de Belleforest vers l'île de Canada, est consacrée à un récit de François de Belleforest, publié en 1572, où l'auteur français réélabore une nouvelle contenue dans l'Heptaméron de Marguerite de Navarre. Il s'agit de la nouvelle de Belleforest "Cœur genereux d'une damoiselle françoise, exposée avec son mary en une isle deserte de l'Ocean, et comme elle en fut delivrée», où est raconté le voyage en mer de deux jeunes qui tombent amoureux et se confrontent à l'agressivité du capitaine de l'expédition. Felice constate que la mer accompagne les vicissitudes des personnages et reflète leurs états d'âme; son statut change au fil du récit, elle représente à la fois le désir, le péril et, en dernière instance, la mort, car le capitaine est une de ses victimes.

5 L'étude de Chiara MOLINARI, Les imaginaires fluvial et maritime au Québec: représentations et stratégies discursives, prend en considération les représentations du Saint-Laurent qui découlent de l'analyse de plusieurs textes d'origine différente. Le but de l'auteure est de comprendre quel portrait du fleuve est proposé à des destinataires différents comme les Québécois et les touristes étrangers. L'analyse des textes adressés aux touristes met en évidence que les premiers aspects évoqués à propos du fleuve sont sa longueur et son aspect majestueux. En même temps, ces textes visent à montrer le Saint-Laurent en tant qu'élément constitutif de l'identité québécoise. En ce qui concerne les textes destinés aux Québécois, ils mettent au premier plan la relation à la fois collective et individuelle qui lie les habitants du Québec au fleuve. À côté des représentations positives, comme la beauté du fleuve et sa biodiversité, dans les textes adressés aux Québécois s'ajoutent d'autres aspects, notamment les problèmes liés à l'érosion ou à l'industrialisation. Selon Molinari, la mise en évidence de ces traits problématiques appartient à une «[...] stratégie visant à la protection du fleuve» (p. 91).

Dans (Ré)écrire le mythe d'Hydro-Québec: "Les Murailles" d'Erika Soucy, Isabelle KIROUAC MASSICOTTE analyse la représentation romanesque des chantiers de l'entreprise publique Hydro-Québec dans Les Murailles (2016) d'Erika Soucy. Le but est de montrer que Soucy revisite un mythe double: le mythe de la colonisation du Nord et le mythe des chantiers d'Hydro-Québec, «[...] nouvelle forme de colonisation de la modernité du Québec» (p. 
97). L'auteure constate que le mythe dont il est principalement question dans Les Murailles, c'est-à-dire celui des chantiers d'Hydro-Québec, s'apparente à une sorte de déconstruction contemporaine $\mathrm{du}$ mythe de la colonisation du Nord. Les hommes qui ont contribué avec leur travail à créer le Québec d'aujourd'hui sont dépeints par Soucy comme des hommes ordinaires, qui vivent souvent un drame personnel, éloignés de leurs proches. Soucy parvient ainsi à «[...] démythifier la colonisation en en révélant les travers et à montrer le Nord comme espace vécu, notamment à partir de la présence autochtone, historiquement occultée» (p. 105).

7 La contribution de Cristina BRANCAGLION, Navigation linguistique et culturelle autour du 'canot', consiste en une étude lexicale du mot «canot» qui vise à illustrer l'importance de ce véhicule dans l'imaginaire des Québécois et la charge culturelle de ses différentes dénominations. Brancaglion s'est basée sur le Fichier Lexical du Trésor de la langue française au Québec, d'où elle a tiré les occurrences du mot «canot». À travers l'analyse des unités lexicales qui définissent les matériaux, la structure et l'usage du canot, Brancaglion a voulu mettre en évidence l'évolution de la charge culturelle attribuée au mot au fil des siècles. Elle a montré ainsi que le canot «[...] s'est rénové en fonction des besoins de chaque époque et demeure un moyen d'interaction avec l'élément liquide en s'intégrant aujourd'hui aux domaines des sports et loisirs» (p. 118).

Prendre souffle dans le limon du fleuve: "Saint-Laurent mon amour" de Monique Durand est le titre de la contribution de Marco MODENESI, qui analyse la présence du fleuve dans le texte de Durand publié en 2017. Saint-Laurent mon amour est un ouvrage hétérogène qui se compose de quatre sections. Modenesi analyse l'évocation des aspects pluriels du fleuve dans le livre, en constatant d'abord que Durand est particulièrement attentive à la description de la flore et de la faune caractéristiques de l'écosystème fluvial. Modenesi souligne ensuite que le regard de Durand s'arrête aussi sur les riverains du passé et du présent, ceux et celles dont les vies sont liées au fleuve. Le mélange de registres et de genres dont Durand fait preuve dans Saint-Laurent mon amour reflète le caractère pluriel de l'univers fluvial. L'importance du livre de Durand réside dans le fait qu'il s'agit, chronologiquement, du dernier texte consacré au Saint-Laurent. Comme l'explique Modenesi, l'auteure de Saint-Laurent mon amour «[...] contribue, de manière extraordinaire, à confirmer, à consolider, à revigorer et à rénover le statut de mythe auquel le Saint-Laurent peut justement aspirer dans l'imaginaire québécois» (p. 128).

9 La dernière étude présentée dans ce numéro de "Ponti/Ponts", Roman de la route et imaginaire du 'Far East' québécois: fleuve, féminité et origines chez Francine Lemay et Noël Audet, signée par David LAPORTE, se focalise sur deux romans où la présence du fleuve et de l'élément maritime renvoie à l'origine, «[...] celle de la fondation du pays et de ses lieux de mémoire, celle, archétypale de la maternité marine» (p. 132). Laporte étudie deux romans de la route où se développe ce qu'il appelle «l'imaginaire de l'Est», Évagabonde de Francine Lemay (1981) et Ah, l'amour l'amour de Noël Audet (1981). L'auteur compare les espaces qui sont représentés dans les romans: le littoral de la Côte-Nord et de la Gaspésie dans Évagabonde et les côtes de la Gaspésie parcourues en auto-stop dans Ah, l'amour l'amour. Les territoires de l'Est québécois, en particulier la Gaspésie, représentent, aux yeux de Laporte, un imaginaire fertile de «[...] mémoire, histoire, fonds de mythes et de légendes» (p. 145).

La section des notes de lecture met fin à ce numéro de "Ponti/Ponts" particulièrement riche du point de vue des contributions proposées, qui dressent un panorama de l'univers maritime au Québec. Malgré la pluralité des perspectives adoptées par les 
auteur.e.s., l'ensemble des textes véhicule un message cohérent ayant pour centre l'élément maritime et son influence sur la culture, l'histoire, la langue et la littérature du Québec. 